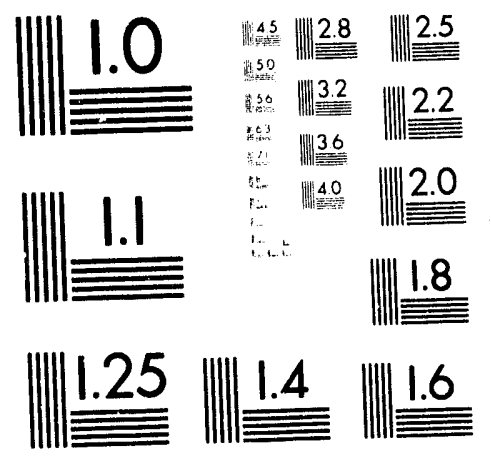



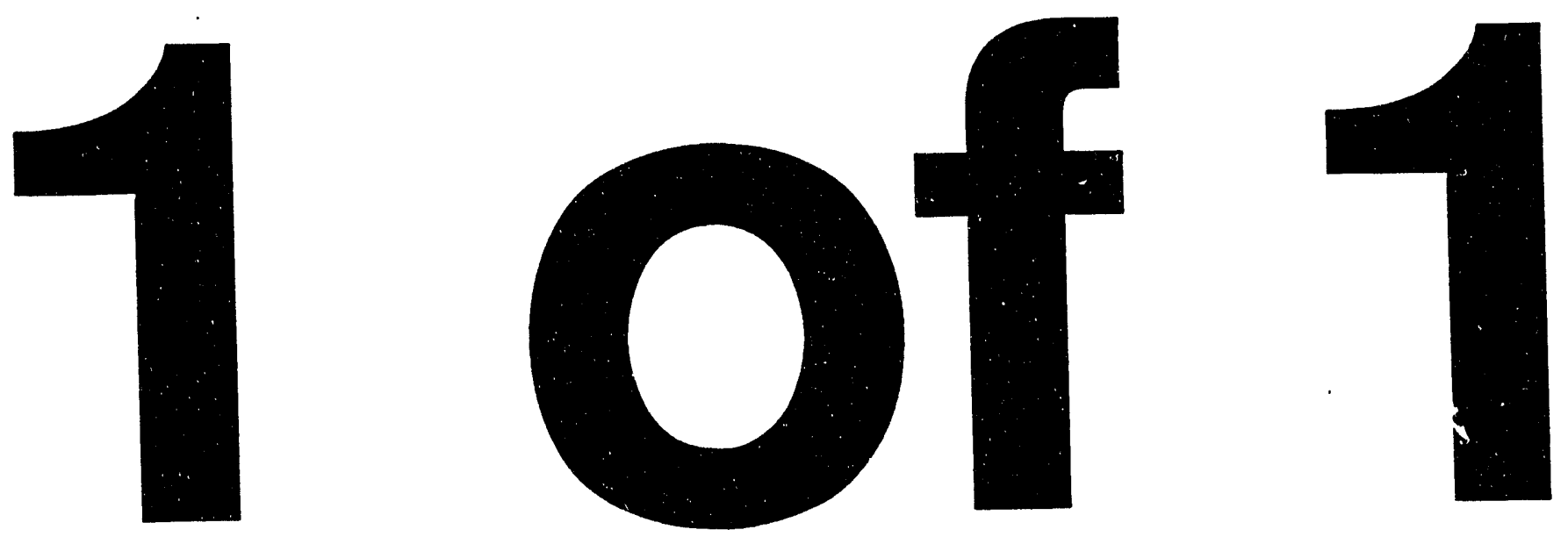

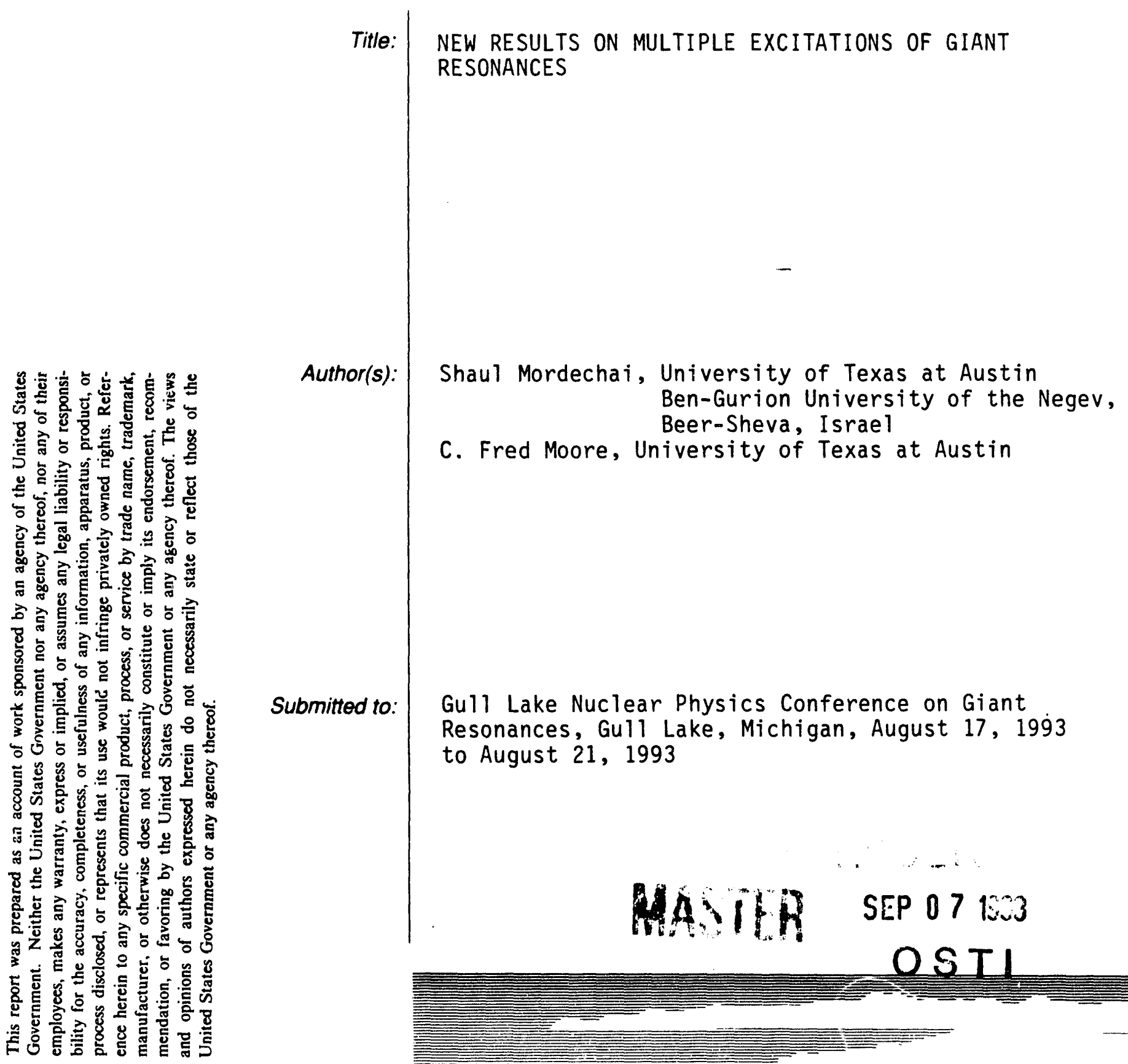

Submitted to:

Gull Lake Nuclear Physics Conference on Giant Resonances, Gul1 Lake, Michigan, August 17, 1993 to August 21, 1993

\section{Los Alamos}

NATIONAL LABORATORY

NEW RESULTS ON MULTIPLE EXCITATIONS OF GIANT 


\title{
New results on multiple excitations of giant resonances
}

\author{
Shaul Mordechai ${ }^{a, b}$ and C. Fred Moore ${ }^{b}$ \\ a Physics Department, Ben-Gurion University of the Negev, Beer-Sheva 84105, Israel \\ bPhysics Department, University of Texas at Austin, RLM 5.208 Austin, Texas, \\ 78712-1081, USA
}

Exotic excitations like the double giant dipole were predicted for many years but not observed experimentally until recently. Several experiments have been carried out at Los Alamos National Laboratory to search for these new collective modes of the nucleus. The results discover two previously unobserved types of double giant resonances. This work presents the recent pion double charge exchange data and the analysis that support the existence of two such exotic vibrational nuclear modes.

\section{INTRODUCTION}

Giant resonances are collective excitations of the nuclear medium induced by external fields that interact with the nuclei by the electromagnetic, weak, or hadronic interaction[1]. Until recently one could study giant resonances only as excitations of the nuclear ground state. An exciting development in nuclear physics in the past decade has been the observation of giant-dipole resonances in highly excited or "hot" nuclei, spinning with high angular momenta. These excitations were predicted by the Brink-Axel hypothesis early in the fifties[2]. The hypothesis assumes that the basic features of the giant resonances (i.e., the frequency of oscillation and the strength of the resonance) are fundamental features governed by the nuclear force and are unaffected by any pre-excitation of the nucleus. Experimental observations to support this hypothesis were made possible only in recent years with the development of heavy-ion accelerators[3]. Results from these studies indicate some remarkable general features of the giant-dipole resonance in excited nuclei when compared with the regular giant dipole built on the nuclear ground state[4].

A special case occurs when the excited state on which the GDR is built is itself a giant resonance. In this case, a collective double giant resonance is excited (i.e., a giant resonance built on another giant resonance). If such excitations do exist, they provide a new test of our understanding of the phenomena of collectivity in nuclei and the basic concepts of giant multipole resonances[5]. The fundamental question is whether a collective mode (GDR or other collective excitations) can still be well formed in special conditions on top of another collective excitation. From a nuclear structure point of view, it is important to understand their properties starting from both macroscopic and microscopic approaches. The macroscopic approach should describe the collective picture of the double giant resonances in terms of multi-nucleon states and the microscopic approach gives the wave functions of these exotic resonances in terms of two-particle, two-hole configura- 
tions. Double resonances in nuclei have not been observed until very recently because of experimental difficulties in observing a resonance located at very high excitation energy, superimposed on a large background from the continuum, and having a large width. In this work we describe the recent results from LAMPF to search for double giant resonances in nuclei.

Figure 1 shows a schematic energy-level diagram of the prominent transitions observable in single charge exchange (SCX) and double charge exchange (DCX). The lowest three double resonances expected in DCX are the double isobaric analog state (DIAS), the giant dipole built on the isobaric analog [or vice versa (GDR $\otimes I A S)$ ], and the double isovector giant-dipole resonance (GDR) ${ }^{2}$. Only the lowest isospin component of the higher two resonances is labeled. The $Q$ values listed in the figure refer to the case of ${ }^{93} \mathrm{Nb}$ discussed later.

An example of pion DCX as an excellent tool to study double resonances is the observation of the well-known DIAS transition $[6,7]$, which can be viewed in this context as the simplest double-resonance state. Until recently $[8,9]$ none of the higher double resonances had been observed. For target nuclei with $J^{\pi}=0^{+}$, the |GDR $\otimes I A S$ ) is a single state with $J^{\pi}=1^{-}$, where $J$ is the total angular momentum and $\pi$ is the

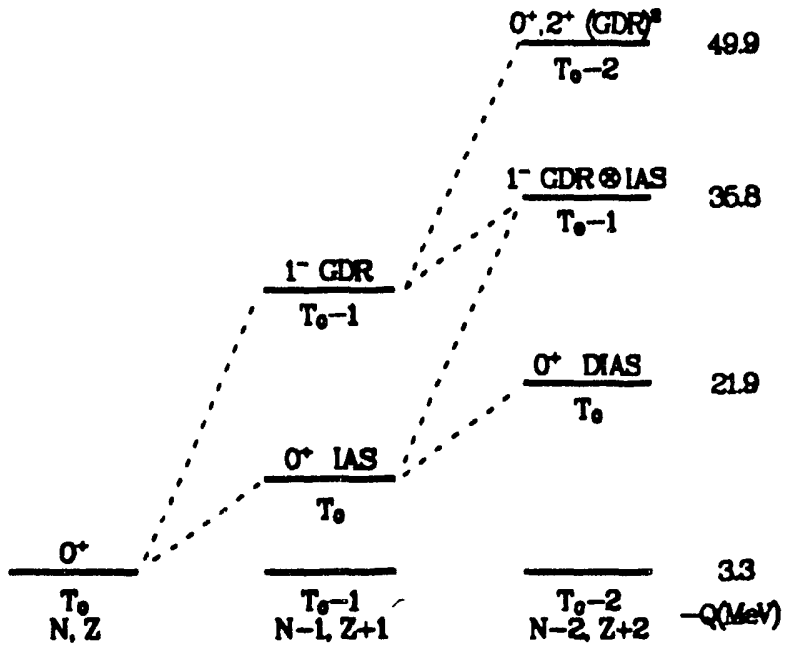

Fig. 1. Schematic energy-level diagram of "single" and "double" resonances anticipated in pion single-charge-exchange $\left(\pi^{+}, \pi^{0}\right)$ and pion double-charge-exchange $\left(\pi^{+}, \pi^{-}\right)$reactions, respectively. The numbers to the right are the $Q$ values for the ground state and the three double resonances observed in the DCX experiment on ${ }^{93} \mathrm{Nb}$.

parity of the state. On the other hand, the double dipole will have two possible spin values, $0^{+}$and $2^{+}$. Both resonances also have different isospin components, but for heavy nuclei with a large neutron excess $(N-Z \gg 1)$, the lowest isospin component (see Fig. 1) is the dominant one.

In the simplest model, pion double-charge-exchange reactions are viewed as two sequential SCX processes in which two neutrons are changed into protons, or vice versa. For target nuclei with $N-Z \geq 1$, the $\left(\pi^{+}, \pi^{0}\right)$ SCX process strongly excites the GDR and the IAS transitions. Therefore, with the high probability for the single-step excitations, a combination of these resonances could, in principle, be reached in DCX. Figure 1 shows the isovector transitions and the energies involved in exciting these new double resonances, as well as the previously well-known double isobaric analog state (DIAS). The DIAS is a prominent transition at forward angles in the $\left(\pi^{+}, \pi^{-}\right)$reaction and has been studied intensively in pion $\operatorname{DCX}[7,10,11]$. 


\section{MEASUREMENTS}

The measurements were performed at the Energetic Pion Channel and Spectrometer (EPICS) at the Los Alamos Meson Physics Facility (LAMPF) using a special setup for pion DCX[12]. The EPICS spectrometer has superior background rejection and precise energy calibration and therefore was most suitable for these studies. The spectrometer can detect outgoing pions with a kinetic energy range of $100-300 \mathrm{MeV}$ with an excellent energy resolution of about, $140 \mathrm{keV}$, and is considered one of the best pion spectrometers in the world. Electrons and muons (arising from pion decay in the channel or within the spectrometer path length) were identified and rejected using a freon-gas Cherenkov detector and a set of scintillators placed behind a series of graphite blocks, respectively[13].

\section{THE GDR BUILT ON THE IAS}

Figure 2 presents typical $Q$-value spectra obtained from pion DCX in a recent experiment at LAMPF to search for double resonances. The figure shows the results on three different targets, ${ }^{13} \mathrm{C},{ }^{27} \mathrm{Al}$, and ${ }^{59} \mathrm{Co}$. The measurements were made at an incident pion kinetic energy of $295 \mathrm{MeV}$ (Ref. [14]). All three spectra show clearly the existence of a wide resonance located in the continuum region at about 8.7 and $14.2 \mathrm{MeV}$ above the ground state of ${ }^{13} \mathrm{O}$ and ${ }^{27} \mathrm{P}$ final nuclei, respectively. On the ${ }^{59} \mathrm{Co}$ target the resonance shows up at about 16.8 $\mathrm{MeV}$ above the DIAS in ${ }^{59} \mathrm{Cu}(27,9 \mathrm{MeV}$ above the ground state of ${ }^{59} \mathrm{Cu}$ ). The resonances are identified as the giant-dipole resonances built on the isobaric analog states or vice versa (GDR@LA.S). The identification is based on their energies, which are very close to the energy where the giant dipoles built on the isobaric analog states are expected to appear, on the characteristic dipole angular distribution of the resonance, and on the cross sections. Similar observations of the resonance have been confirmed on many other nuclei, indicating

Fig. 2. $Q$-value spectra for $\left(\pi^{+}, \pi^{-}\right)$pion double-chargeexchange reactions at $\mathrm{T}_{\pi}=295 \mathrm{MeV}$ on: $(\mathrm{a}){ }^{13} \mathrm{C}$ at $\theta_{\text {lab }}=$ $18^{\circ}$, (b) ${ }^{27} \mathrm{Al}$ at $\theta_{l a b}=15^{\circ}$, and (c) ${ }^{59} \mathrm{Co}$ at $\theta_{l a b}=11^{\circ}$. The arrows indicate the fitted location of the ground state, the DIAS, and the giant resonance (GDROIAS). Short vertical lines represent statistical uncertainties of the data. The dashed lines are the fitted backgrounds with a polynomial shape, and the solid lines are the fits to the spectra using a line-shape fitting code NEWFIT.

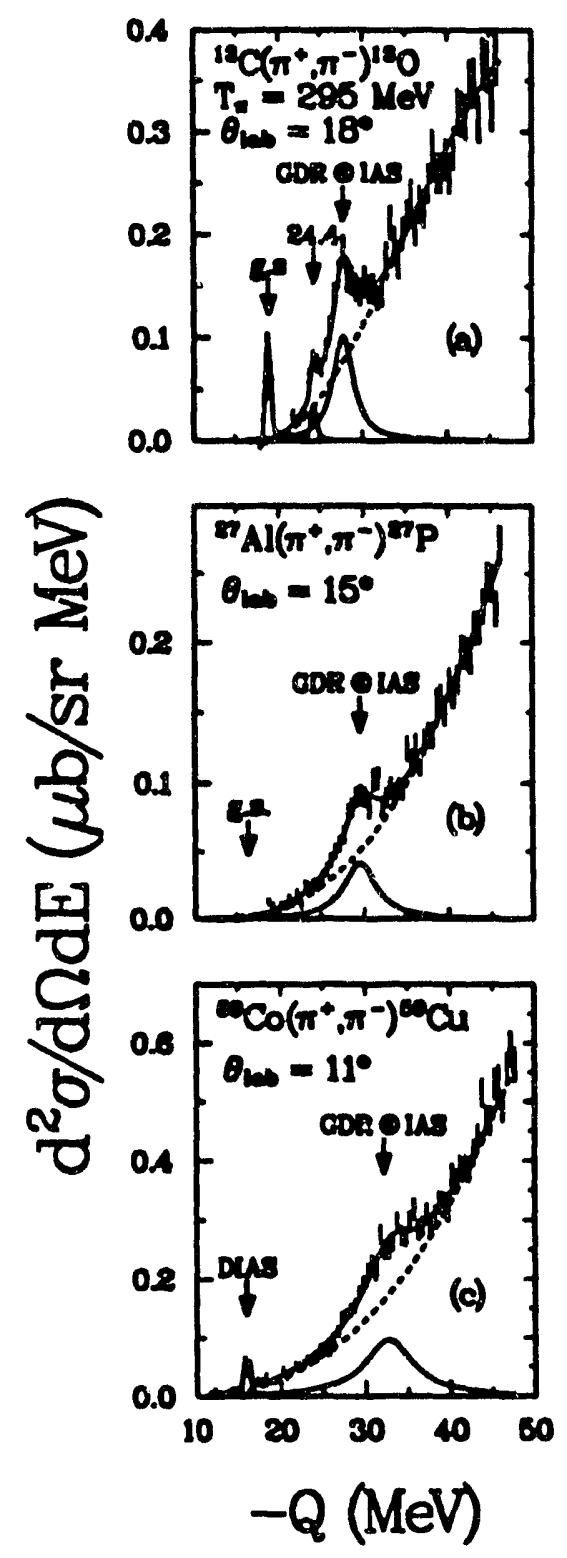


that this collective mode is a general feature of all nuclei that have at least one excess neutron[15]. The ground state, the DIAS and the resonances were fitted with a standard peak shape. The background (dashed lines), which arises from DCX to the continuum, was fitted using a third-order polynomial shape. The energies and widths were varied simultaneously to minimize $\chi^{2}$ for the entire fit. The figure shows clearly that the width of the GDR $\otimes I A S$ increases significantly with mass. For example, the resonance on ${ }^{13} \mathrm{C}$ has a width of $3.0 \pm 0.6 \mathrm{MeV}$, but on ${ }^{59} \mathrm{Co}$ the resonance has a width of $7.0 \pm 1.0 \mathrm{MeV}$ (i.e., more than twice as wide as that on ${ }^{13} \mathrm{C}$ ). The increase of the width with mass is not yet fully understood. This is one of the puzzles arising from these studies. Further details on these resonances can be found in Ref. [15], where some systematic features of the GDR $\otimes I A S$ are outlined. The results indicate that in many respects (e.g., energies, cross sections, and variations of the cross sections with mass) the GDR $\otimes$ IAS does behave as a superposition of the two-ingredient resonances, the GDR and the IAS.

Figure 3 shows the ${ }^{93} \mathrm{Nb} Q$-value spectra at three angles. In addition to the DIAS at $\mathrm{E}_{x}=19.3 \mathrm{MeV}(Q=-21.9$ $\mathrm{MeV}$ ), the spectra show the existence of a relatively wider peak located in the continuum region at about $13.9 \mathrm{MeV}$ above the DIAS. This peak is labeled GDR $\otimes I A S$ (GR1) in the figure. The DIAS is strong at $5^{\circ}$ but very weak at $10^{\circ}$ and $20^{\circ}$, whereas GR 1 , has a maximum around $10^{\circ}$. The peaks were fitted with a Gaussian and a Lorentzian shape of variable widths for the DIAS and GR1 respectively. In Fig. 3 we show a fit to the spectra with $\Gamma_{D I A S}=0.8$ $\mathrm{MeV}$ and $\Gamma_{G R 1}=5.8 \mathrm{MeV}$. The spectra can be well fitted also using a Gaussian line shape for the GR, resulting in a smaller cross section by about $50 \%$. The width of the DIAS arises primarily from resolution energy due to the thick $\left(1.714 \mathrm{~g} / \mathrm{cm}^{2}\right){ }^{93} \mathrm{Nb}$ target. The spectra have been corrected for the spectrometer acceptance as a function of momentum. The background (dashed line) that arises from DCX to discrete low-lying states and to the continuum was fitted using a third-order polynomial of the form $R(1)+R(2) \times E+R(3)$ $\times E^{2}+R(4) \times E^{3}$ where $E$ is the abso-

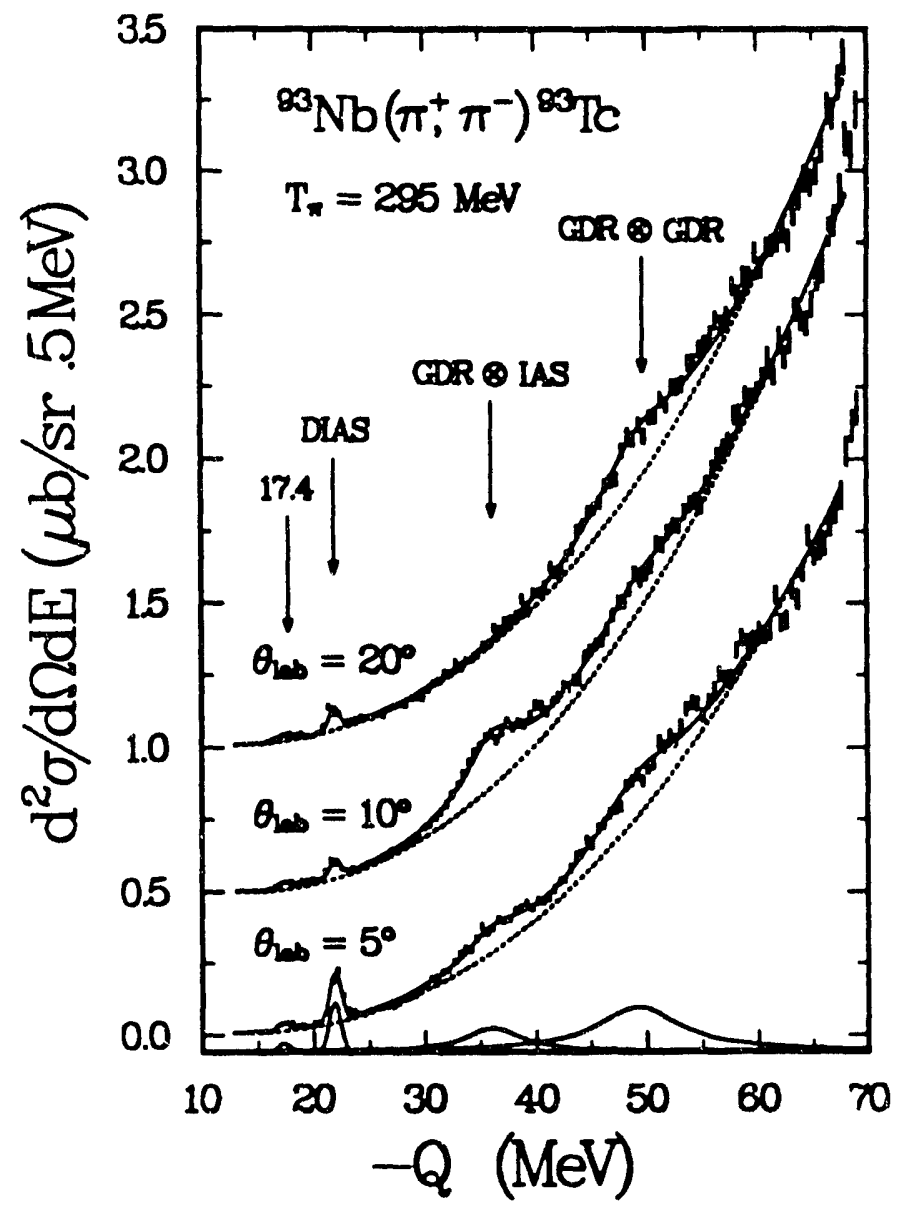

Fig. 3. Doubly differential cross section for the $\left(\pi^{+}, \pi^{-}\right)$reaction on ${ }^{93} \mathrm{Nb}$ target at $\mathrm{T}_{\pi}=$ $29.5 \mathrm{MeV}$ and $\theta_{l a b}=5^{\circ}, 10^{\circ}$, and $20^{\circ}$. The arrows indicate the three double resonances observable in pion DCX: (1) the DIAS, (2) the

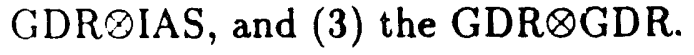


lute $Q$ value. It was found that comparable fits and cross sections are obtained if, instead, fourth- or higher-order polynomial shapes are used for the background. The solid line is the resulting fit to the spectrum. The upper resonance in Fig. 3 labeled GDR $\otimes$ GDR will be discussed later.

Figure 4 presents the angular distribution extracted for GR1 on ${ }^{93} \mathrm{Nb}$ at $295 \mathrm{MeV}$. The maximum cross section is observed at $10^{\circ}$ with a smaller cross section at $5^{\circ}$ and $15^{\circ}$. At scattering angles of $20^{\circ}-25^{\circ}$ the resonance is very weak and can hardly be observed, but at $30^{\circ}$ the peak shows up again above background. The solid and dashed lines in Fig. 4 are simple calculations in which sequential single charge exchange through the isobaric analog to the giant dipole resonance is evaluated with two different transition densities for the IAS[15]. The collective model has been used to obtain the radial shape of the transition densities for the dipole. The calculated cross sections using both models reproduce the dipole shape of the data and account surprisingly well for the measured cross sections.

\subsection{Energy dependence of the GDR $\otimes$ IAS}

Figure 5 shows the ${ }^{13} \mathrm{C}\left(\pi^{+}, \pi^{-}\right)^{13} \mathrm{O}$ missing-mass spectra at five different incoming pion beam energies. The scattering angles have been chosen to correspond to the peak cross section of the giant dipole built on the isobaric analog state. In addition to the ground state (gs) and the three lowlying narrow states reported earlier[16] (at $Q=$ $-22.0 \mathrm{MeV}, Q=-23.4 \mathrm{MeV}$, and $Q=-25.0$ $\mathrm{MeV}$ ), most spectra show clearly tbe existence of

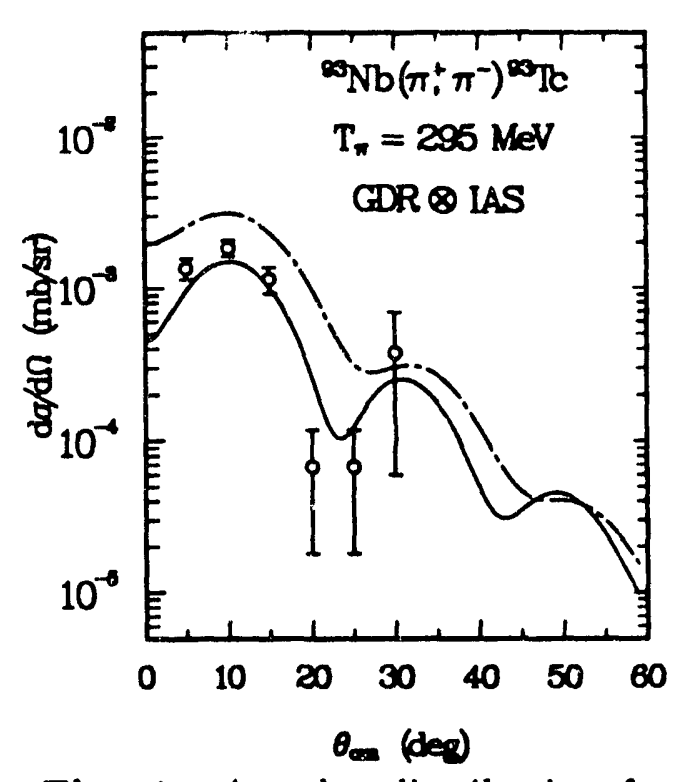

Fig. 4. Angular distribution for the peak labeled GDRQIAS in the double-charge-exchange spectrum on ${ }^{93} \mathrm{Nb}$ shown in Fig. 3. The cross sections have been extracted with use of a Lorentzian line shape for the giant resonance with constant width of $5.8 \mathrm{MeV}$ and constant $Q=-35.8 \mathrm{MeV}\left(\mathrm{E}_{x}=33.2\right.$ $\mathrm{MeV})$. The solid and chain-dashed lines are sequential model calculations using volume and surface transition density, respectively. a wider peak located in the continuum region at $Q=-27.6 \mathrm{MeV}\left(E_{x}=8.7 \mathrm{MeV}\right.$ above the gs of $\left.{ }^{13} \mathrm{O}\right)$. This resonance was earlier identified as the $T_{>}=3 / 2$ component of the giant dipole resonance built on the isobaric analog state[14] (see Fig. 2). In Fig. 5, this resonance is labeled (GDR $\otimes I A S)$. For $T=1 / 2 \mathrm{nu}$ clei, the $T_{<}=1 / 2$ component of the (GDR $\left.\otimes I A S\right\rangle$ is forbidden. The ground state and the low-lying narrow states were fitted with a Gaussian, and the resonance with a Lorentzian shape of variable widths. Figure 5 demonstrates that the cross section for the |GDR $\otimes I A S\rangle$ increases significantly with beam energy. The resonance shows up clearly in the $295-\mathrm{MeV}$ spectrum, but almost vanishes in the $140-\mathrm{MeV}$ measurement. The transitions to the three lower excited states exhibit a substantially different excitation function. Their cross sections decrease with increasing beam energy. These states have been observed earlier[16], but little is known of their nature. 


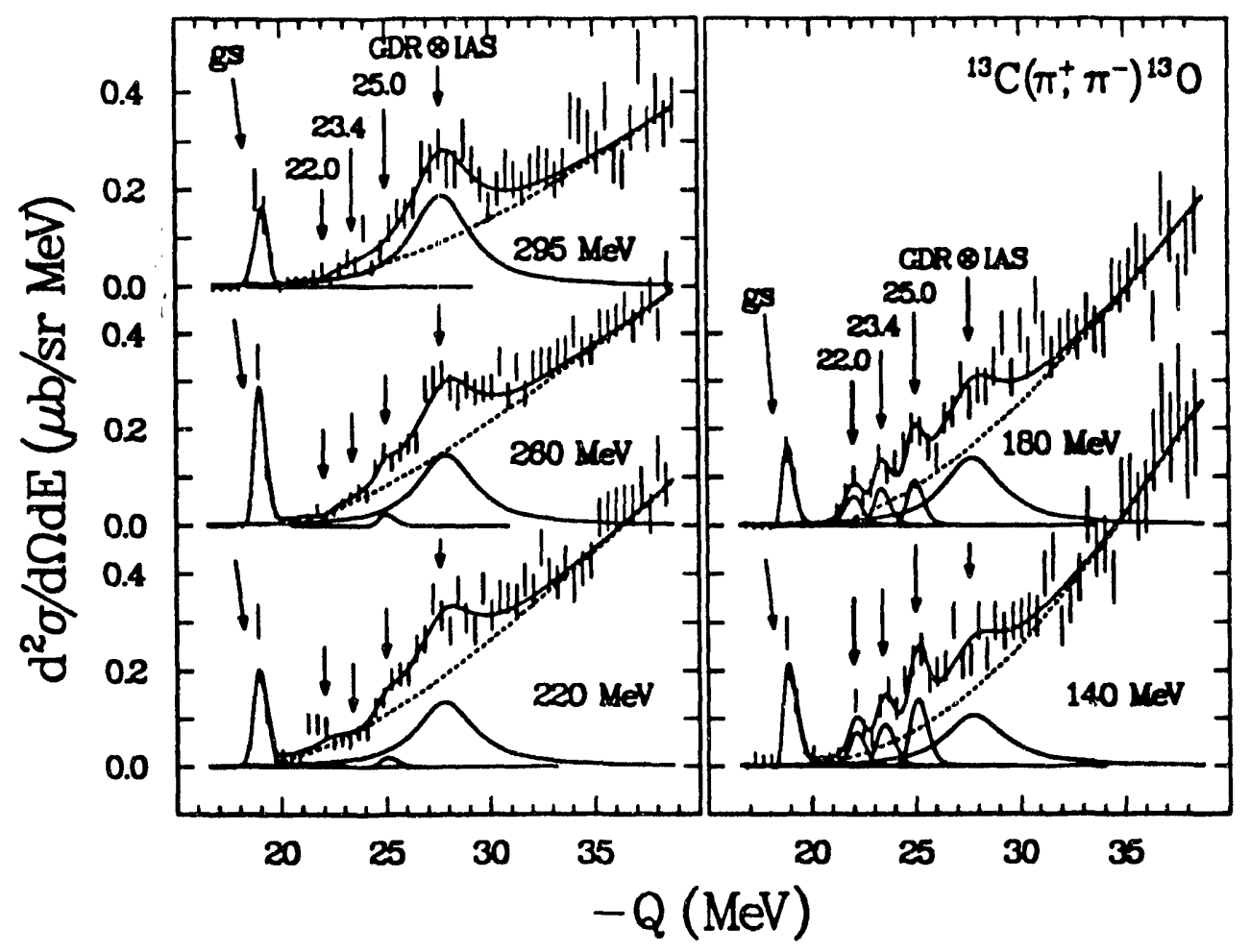

Fig. 5. Double-differential-cross section spectra for the $\left(\pi^{+}, \pi^{-}\right)$reaction on a ${ }^{13} \mathrm{C}$ target at $\mathrm{T}_{\pi}=295,260,220,180$, and $140 \mathrm{MeV}$. The arrows indicate the fitted location of the gs, the three low-lying states, and the giant resonance. Short vertical lines represent statistical uncertainty of the data. The dashed lines are the fitted background with a polynomial shape and the solid lines are the fits to the spectra using NEWFIT.

In order to understand the observed energy dependence in a simple model, we can use an expression for the energy dependence of the inelastic cross section derived by Siciliano and Walker [17] in a strong absorption model assuming the dominance of the $[3,3]$ resonance:

$\sigma(\theta)=\alpha(E)\left[4 M^{2}\left(q_{0}\right) \cos ^{2}(\theta)+S^{2}\left(q_{0}\right) \sin ^{2}(\theta)\right]$.

Here, $q_{c}$ is a constant momentum transfer near the maximum of the differential cross section, and $\alpha(E)$ contains the energy dependence of the elementary $\pi-N$ force. In general, only the $S$ form factor can contribute to $\Delta S=1$ spin transitions. The equation indicates that the $\Delta S=0$ non-spin-flip transition cross section increases with energy like $\cos ^{2}(\theta)$ at a constant momentum transfer since $q_{0}=2 k \sin (\theta / 2)$, where $k$ is the beam momentum. Using the same argument, the spin-flip transitions should decrease with energy like $\sin ^{2}(\theta)$. This technique for distinguishing spin-flip from non-spin-flip transitions by examining the constant- $q$ excitation function was successfully used earlier on ${ }^{13} \mathrm{C}$ [18], to identifv $\Delta S=0$ and $\Delta S=1$ transitions in pion scattering. Calculation of the |GDR $\otimes$ IAS $\rangle$ with a fixed transition strength for the GDR and the IAS gives an adequate representation of the measured excitation function for this resonance. The analysis [27] indicates that the excitation of the $\mid$ GDR $\otimes I A S$ ) in this energy region proceeds by a two-step sequential 
$\left(\pi^{+}, \pi^{0}\right)\left(\pi^{0}, \pi^{-}\right)$mechanism and, therefore, pion DCX should be a unique probe for exciting isotensor double giant resonances in nuclei.

\section{THE DOUBLE ISOVECTOR GIANT DIPOLE RESONANCE}

Figure 3 presents $Q$-value histograms measured for the ${ }^{93} \mathrm{Nb}\left(\pi^{+}, \pi^{-}\right)^{93} \mathrm{Tc}$ reaction at $295 \mathrm{MeV}$ at three different scattering angles. These spectra were taken in long DCX measurements in order to accumulate good statistics. They also cover a high excitation energy. The $|G D R \otimes I A S\rangle$ has been discussed in section 3. The peak labeled GDR $\otimes$ GDR (GR2) has a different angular distribution. Full angular distributions for the resonances have been measured on ${ }^{40} \mathrm{Ca},{ }^{93} \mathrm{Nb}$, and ${ }^{56} \mathrm{Fe}$ (Ref. [19]). Detailed analysis shows that the two resonances have different natures since they have a significantly different angular dependence. The GDR $\otimes$ IAS has a dipole shape. The higher resonance GR2 has a quadrupole distribution. Based on its energy (which is about twice the energy of the one-step GDR), angular distribution, and cross section, we identify GR2 as the double giant dipole[19]. The double dipole has a width of about $8-10 \mathrm{MeV}$, which is larger than the width of the "single" dipole by a factor of $1.5 \rightarrow 2.0$. This was found to be in good agreement with theoretical estimates for the width of the double dipole in terms of two phonon states[19-21].

For heavy nuclei, a simple relation is expected between the three double resonances observed in DCX, arising from the addition of one- and two-giant-dipole energies as illustrated in Fig. 1:

$\left|Q_{\mathrm{GDR} \otimes \mathrm{IAS}}\right|-\left|Q_{\mathrm{DIAS}}\right|=\left|Q_{\mathrm{GDR}}\right|-\left|Q_{\mathrm{GDR} \otimes \mathrm{IAS}}\right|$.

The experimental energies are in close agreement with the above simple relation, which gives additional support for the proper identification of the resonances. Experimentally the single-charge-exchange GDR is observed in $\left(\pi^{+}, \pi^{0}\right)$ around $Q=-25 \mathrm{MeV}$ for a wide range of mass. Thus, the energies of the double dipoles observed in $\left(\pi^{+}, \pi^{-}\right)$are close to double the energy of the "single" GDR, i.e.,

$Q_{\pi^{+}, \pi^{-}}\left(G D R^{2}\right) \approx 2 \times Q_{\pi^{+}, \pi^{0}}(G D R) \approx-50 \mathrm{MeV}$.

This near equality is surprising because it neglects the effect of isospin splitting, which exists in both the $\left(\pi^{+}, \pi^{0}\right)$ and the $\left(\pi^{+}, \pi^{-}\right)$reactions, but is somewhat different in the two reactions.

A unique test for the identification of the double dipole can be provided by measuring the inverse reaction, i.e., the $\left(\pi^{-}, \pi^{+}\right) \Delta T_{z}=+2$ PCX mode $\mathrm{ga}$ the same target nuclei. Figure 6 shows the ${ }^{40} \mathrm{Ca}\left(\pi^{-}, \pi^{+}\right)^{40} \mathrm{Ar}$ and the $\left.{ }^{40} \mathrm{Ca}, \pi^{-}\right)$If $^{-} Q$-value spectra measured under the same experimental conditions at $\theta_{\text {lab }}=5^{\circ}$ and coming pion energy $\mathrm{T}_{\pi}=295 \mathrm{MeV}$. In addition to the weak transitions to the ground dtate both spectra contain a wide peak labeled (GDR) $)^{2}$ located in the continuum region. In the $\left(\pi^{+}, \pi^{-}\right)$ reaction, the resonance is observed[19] at $Q=-54.0 \mathrm{MeV}$, but in the inverse reaction, $\left(\pi^{-}, \pi^{+}\right)$, the resonance appears at a much lower energy around $Q=-31.1 \mathrm{MeV}$. The GR peaks were fitted with a Lorentzian shape of variable width. The fits shown in Fig. $\dot{0}$ use $\Gamma(\mathrm{GDR})^{2}=9.0 \mathrm{MeV}$ for both reactions. The spectra have been corrected for the 
spectrometer acceptance as a function of momentum. The background (dashed line), which arises from the DCX cross section to discrete low-lying states, and to the continuum, was fitted using a third-order polynomial function of the $Q$-value. The resonance in the ${ }^{40} \mathrm{Ca}\left(\pi^{+}, \pi^{-}\right)^{40} \mathrm{Ti}$ data was identified[19] as the double isovector giantdipole resonance based on its energy, characteristic angulas distribution, and cross section (Fig. 7). The double dipole reached in the $\left(\pi^{-}, \pi^{+}\right)$reaction appears at almost exactly four Coulomb displacement energies (minus four neutron-proton mass differences), as expected. In $\left(\pi^{+}, \pi^{-}\right)$, the double GDR appears around $Q=-50$ $\mathrm{MeV}$ and its energy is almost independent of $A$. The doubly excited GDR in the $\left(\pi^{+}, \pi^{-}\right)$reaction is reached by adding two E1 vibrational energies and two Coulomb energies to the target nucleus ground state. The E1 energy decreases with $A$, but the Coulomb energy increases with $Z$ and the two effects almost cancel each other, yielding a nearly constant $Q$ value for the double GDR excitations. The double GDR observed in $\left(\pi^{-}, \pi^{+}\right)$has a significantly different trend. This state is obtained by adding two $E 1$ vibrational energies to the nucleus and subtracting two Coulomb energies, as two protons are changed into two neutrons. Thus, the $Q$ values in $\left(\pi^{-}, \pi^{+}\right)$ are expected to decrease strongly (in absolute value) with $A$. The lowering of the magnitude of the double-dipole $Q$ value is very significant for medium and heavy nuclei. For example, the double dipole on ${ }^{93} \mathrm{Nb}$ has $Q=-49.9 \mathrm{MeV}$ in ${ }^{93} \mathrm{Nb}\left(\pi^{+}, \pi^{-}\right){ }^{93} \mathrm{Tc}$ but appears as low as $Q=-24.9 \mathrm{MeV}$ in the ${ }^{93} \mathrm{Nb}\left(\pi^{-}, \pi^{+}\right)^{93} \mathrm{Y}$ reaction.

\section{1. Angular distributions and theoretical calculations}

Figure 7 (a) presents the angular distribution extracted for the GR peak on ${ }^{40} \mathrm{Ca}$. Figures 7 (b) and 7 (c) show the angular distribution for both GR1 and GR2 on ${ }^{93} \mathrm{Nb}$ and ${ }^{56} \mathrm{Fe}$, respectively. The two resonances, GR1 (lower) and GR2 (upper), shown in Fig. 3 , have different angular distributions. GRl peaks around $10^{\circ}$ and has a dipole shape, 
whereas the higher resonance (GR2) has a quadrupole distribution that is similar in shape and magnitude to the single GR peak observed on ${ }^{40} \mathrm{Ca}$. We identify GR1 on ${ }^{56} \mathrm{Fe},{ }^{93} \mathrm{Nb}$, and several other $\mathrm{T} \geq 1$ targets as the giant dipole built on the isobaric analog state, GDR $\otimes I A S$, discussed earlier. This resonance is absent on ${ }^{40} \mathrm{Ca}$ (and other self-conjugate targets) because no IAS, and hence no GDR $\otimes I A S$, is possible on a $T=0$ nucleus.

A simple sequential model has been used to predict the cross section and angular distribution for the doubledipole state. We used the pion coupled-channel impulse approximation code (CCIA) NEWCHOP[22]. The calculations for the double dipole include the ground state (gs), the giant dipole resonance (GDR), and the double dipole $\left(\mathrm{GDR}^{2}\right)$. A widely accepted collective Tassie form factor for the transition density, $r^{L-1} \rho^{\prime}(r) \mathcal{Y}_{L M}(\hat{r})$, with $L=1$ was used for the GDR[23], where $\rho(r)$ is the nuclear ground state density. The strength of each SCX has been adjusted to reproduce the measured or extrapolated $\left(\pi^{+}, \pi^{0}\right)$ cross section for the GDR at 165 $\mathrm{MeV}[24]$. The transition strength thus obtained was used at $295 \mathrm{MeV}$ for both SCX processes. This method was tested against SCX data[25] at lower energies. Further details on the calculations for the double dipole can be found in Refs. [19] and [26].

Fig. 7 (a) Angular distribution for the peak labeled GR in the double-charge-exchange spectra on ${ }^{40} \mathrm{Ca}$ shown in Fig. 6. The cross sections have been extracted with use of a Lorentzian line shape for the giant resonance with constant width of $9.0 \mathrm{MeV}$ and constant $Q=-54.0$ $\mathrm{MeV}$. The solid curve is from a sequential-model calculation for the double-dipole $\left(J^{\pi}=2^{+}\right)$state, but renormalized as mentioned in Ref. [19]. The chain-dashed curve for the double-dipole $\left(J^{\pi}=0^{+}\right)$state is shown without any normalization factor. (b) and (c) The same as (a) except for ${ }^{93} \mathrm{Nb}\left(\pi^{+}, \pi^{-}\right)$and ${ }^{56} \mathrm{Fe}\left(\pi^{+}, \pi^{-}\right)$reactions. The solid and chain-dashed lines are the theoretical calculations for the double dipole and the dipole built on the isobaric analog state, respectively.

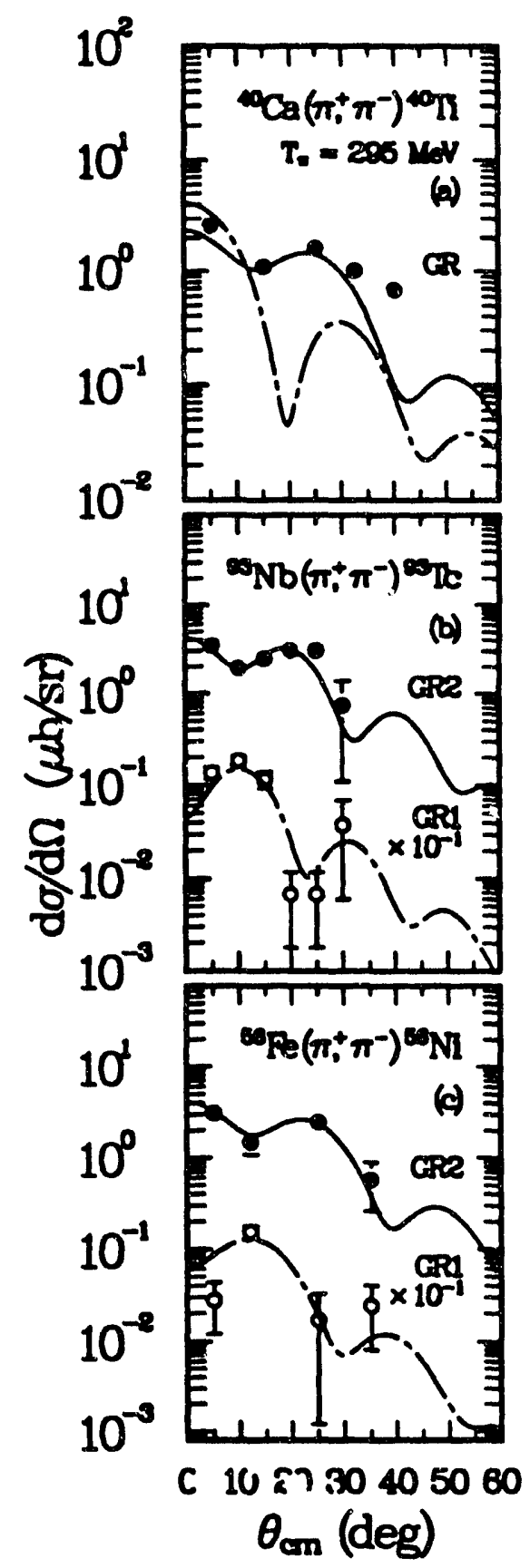




\section{Acknowledgements}

We wish to acknowledge the substantial assistance in the performance of these experiments given by many people at the University of Texas at Austin, The University of Pennsylvania, New Mexico State University, The University of York, and Los Alamos National Laboratory. Experiments have been done from 1984 through 1992 by a large collaboration whose members changed with time. Many discussions with N. Auerbach, D. Bowman, H. T. Fortune, B. Gibb; , M. Johnson, and C. Morris are gratefully acknowledged. We also thank Herb Ward and Charles Whitley for assistance in preparing this manuscript. This work is supported by the U. S.-Israel Binational Science Foundation, the Robert A. Welch Foundation, and the U. S. Department of Energy.

1. M. Goldhaber and E. T'eller, Phys. Rev. 74, 1046-1049 (1948).

2. D. M. Brink, Ph. D. Dissertation, University of Oxford, 1955 (unpublished); P. Axel, Phys. Rev. 126, 671-683 (1962).

3. G. F. Bertsch and R. A. Broglia, Physics Today 39, \#8, August (1986).

4. K. A. Snover, Ann. Rev. Nucl. Part. Sci. 36, 545-603 (1986) and references therein.

5. N. Auerbach, Proceedings of the Workshop on Pion-Nucleus Physics: Future Directions and New Facilities at LAMPF, AIP Conference Proceedings 163, 34-53, Los Alamos, 1987.

6. R. Gilman, Proceedings of the LAMPF Workshop on Pion Double Charge Exchange, Los Alamos National Laboratory Report No. LA-10550-C, p. 14-44, 1985 (unpublished).

7. M. B. Johnson and C. L. Morris, Annual Review of Nuclear and Particle Sciences (in press) (1993), and references therein.

8. S. Mordechai et al., Phys. Rev. Lett. 60, 408-411 (1988).

9. S. Mordechai et al., Phys. Rev. Lett. 61, 531-534 (1988).

10. W. R. Gibbs and M. J. Leitch, Pion-Nucleus Double Charge Exchange, Singapore: World Scientific (1990).

11. H. Clement, Prog. Part. Nucl. Phys. 29, 175-250 (1992).

12. H. A. Thiessen et al., Los Alamos Scientific Laboratory Report No. LA-6663-MS (1977) (unpublished); S. J. Greene et al., Phys. Lett. 88B, 62 (1979).

13. C. L. Morris et al., Nucl. Instrum. \& Methods A 238, 94 (1985).

14. S. Mordechai et al., Phys. Rev. C 43, 1111 (1991).

15. S. Mordechai et al., Phys. Rev. C 40, 850-858 (1989).

16. S. Ajzenberg-Selove, Nuc. Phys. A523, 1 (1991).

17. E. Siciliano and G. E. Walker, Phys. Rev. C 23, 2661 (1981).

18. S. Seestrom-Morris et al., Phys. Rev. Lett., 46, 1447 (1981).

19. S. Mordechai et al., Phys. Rev. C 41, 202-212 (1990).

20. N. Auerbach, Ann. Phys. 197, 376-395 (1990).

21. Ph. Chomas and N. Van Giai, Phys. Lett. B 282, 13 (1992).

22. E. Rost, computer code CHOPIN (unpublished). The code has been modified by C. L. Morris to calculate pion charge exchange reactions and renamed NEWCHOP.

23. L. J. Tassie, Aust. J. Phys. A305, 509 (1978).

24. A. Erell, J. Alster, J. Lichtenstadt, M. A. Moinester, J. D. Bowman, M. D. Cooper, F. Irom, H. S. Matis, E. Piasetzky, and U. Sennhauser, Phys. Rev. C 34, 1822 (1986).

25. F. Irom et al., Phys. Rev. C 34, 2231 (1986).

26. H. Ward et al., Phys. Rev. C 45, 2723 (1992).

27. H. Ward et al., Phys. Rev. Lett. 70, 3209 (1993). 

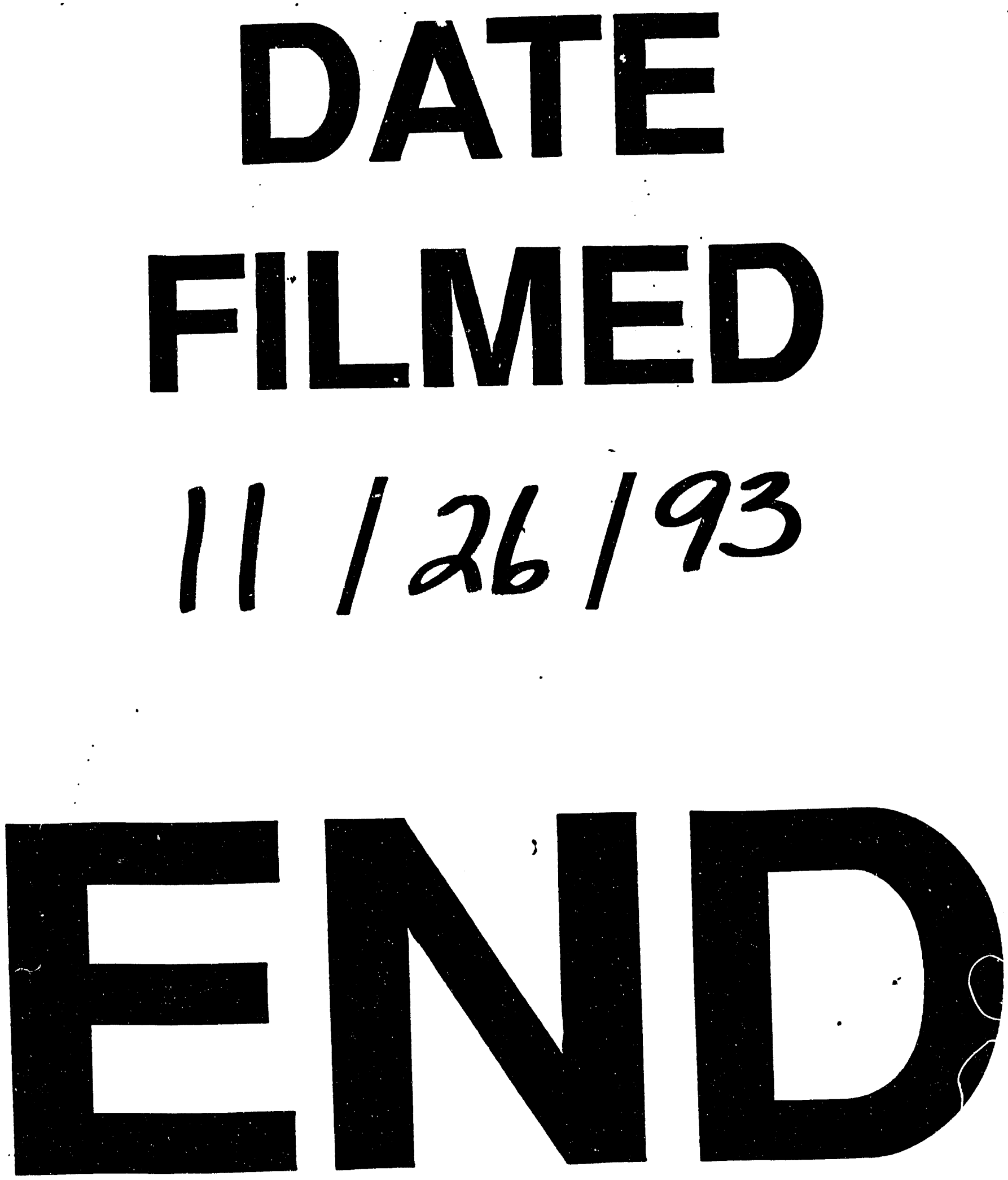
